\title{
Wie man Ausbildungsabbrüche vermeiden kann
}

\section{Problembeschreibung und Möglichkeiten der Prävention}

\author{
Andreas Frey und Philipp Terhart
}

Prof. Dr. Andreas Frey, Berufs- und Wirtschaftspädagoge, lehrt und forscht an der Hochschule der Bundesagentur für Arbeit im Bereich der

Kompetenzdiagnostik, Kompetenzberatung, Qualifikationsund Curriculumsentwicklung.

E-Mail

Andreas.Frey2@arbeitsagentur.de

Philipp Terhart ist Berufsberater und arbeitet bei der Agentur für Arbeit in Essen.

E-Mail p.terhart@gmx.de
Seit Jabren ist die Abbrecherquote in der Berufsausbildung in Europa konstant auf hohem Niveau. Dies betrifft nicht nur die Länder mit dualem Ausbildungssystem, sondern auch diejenigen mit vornehmlich schulischer Berufsbildung. Ein Ausbildungsabbruch hinterlässt meist bei allen beteiligten Personen ein Gefühl des Scheiterns und birgt sowohl erwerbsbiografische als auch sozial- und arbeitsmarktpolitische Risiken. Bei den beteiligten Betrieben, vor allem den kleinen und mittleren Unternehmen, hinterlässt ein Abbruch oft nicht nur wirtschaftlichen Schaden, sondern auch abnehmende Ausbildungsbereitschaft.

Viele innovative Maßnahmen und Strategien verschiedener europäischer Länder belegen, dass der Ausbildungsabbruch ein aktuelles Thema ist. Es fällt aber auf, dass der Fokus mehrheitlich auf Maßnahmen und Strategien nach Ausbildungsabbruch gerichtet ist. Case Management, Betreuungsdienste und weitere Angebote, die Ausbildungsabbrecher auffangen helfen, sowie Werkzeuge und Prozesse, die Jugendliche zur Wiederaufnahme einer Ausbildung bewegen wollen, sind in fast allen Ländern zu finden.

Präventivmaßnahmen, die Jugendliche bereits während der Schulpflicht auf die weiterführenden Ausbildungen vorbereiten, oder im ersten Ausbildungsjahr potenzielle Ausbildungsabbrecher gezielt er-

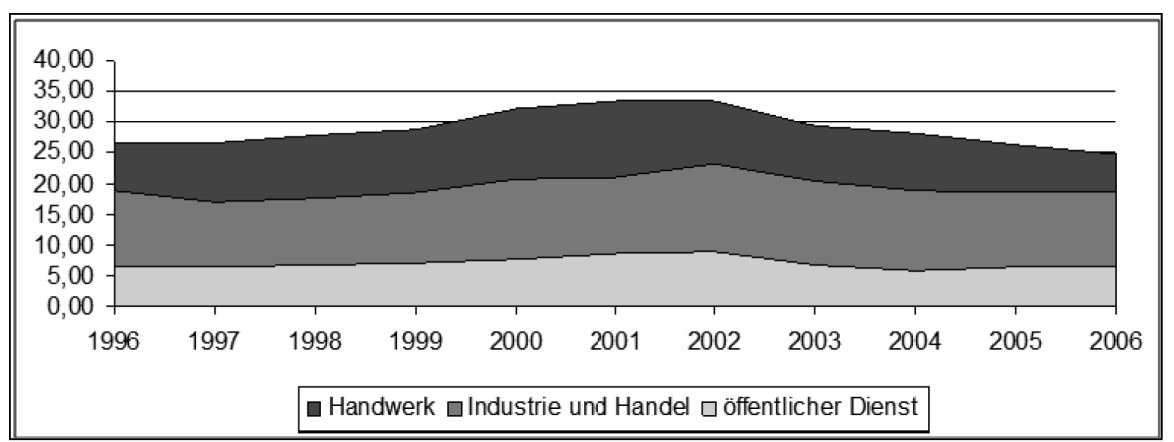

Abb. 1: Entwicklung der Abbrecherquote von 1996 bis 2006

Quelle: Statistisches Bundesamt fassen, sind selten. Nur Irland und Dänemark versuchen, alle gefährdeten Jugendlichen vor Ausbildungsabbruch zu diagnostizieren, und Norwegen zielt mit dem Schulfach »Educational Choises « auf eine breite Sensibilisierung der Jugendlichen für unterschiedliche Ausbildungen und deren Anforderungen. Eine »Dropout «Quote von unter zehn Prozent belegt insbesondere für Dänemark und Finnland, wie effektiv Präventivmaßnahmen sein können. (1)

\section{Problembeschreibung}

In Deutschland liegt die Abbrecherquote im Durchschnitt bei etwa 20 Prozent (siehe Abb. 1), wobei die Prozentzahlen zwischen den Branchen stark variieren: So betragen im öffentlichen Dienst durchschnittlich 7 Prozent, in Industrie und Handel 19 Prozent und im Handwerk 25 Prozent. (2) Die Abbrecherquote sagt nichts darüber aus, wer den Ausbildungsvertrag letztendlich gelöst hat, der Betrieb oder der Jugendliche. Die Abbrecherquote liefert ebenso keine Informationen darüber, in welche qualitative Richtung eine Ausbildung vom Jugendlichen abgebrochen wurde.

Je nach Verbleib des Jugendlichen nach einer Vertragsauflösung unterteilt man sie in drei Gruppen (siehe Abb. 2). Ein »Abbruch nach oben « bedeutet, dass 


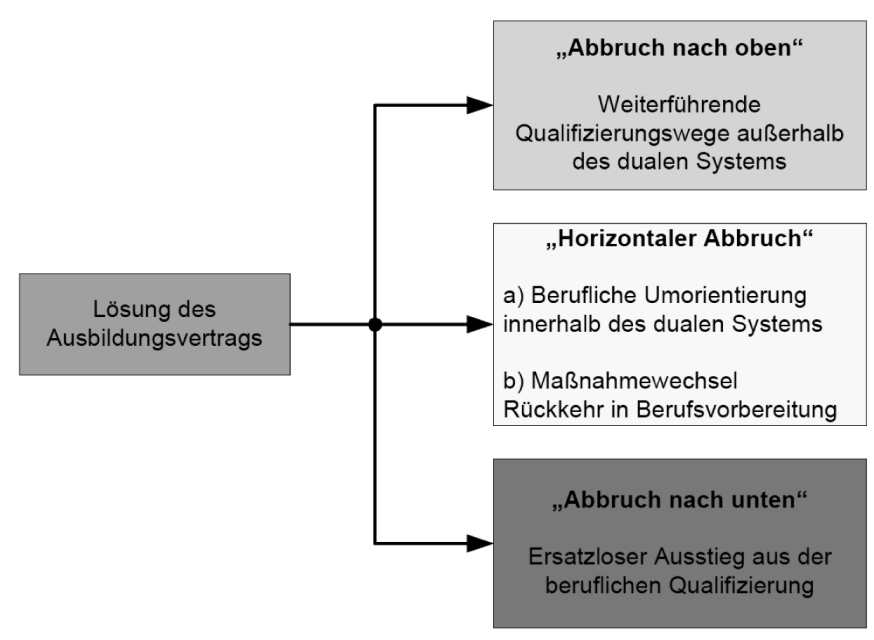

Abb. 2: Arten von Ausbildungsabbrüchen

Quelle: Faßmann, H. (1998). Das Abbrecherproblem - die Probleme der Abbrecher. Zum Abbruch der Erstausbildung in Berufsbildungswerken. Nürnberg: Institut für empirische Soziologie.

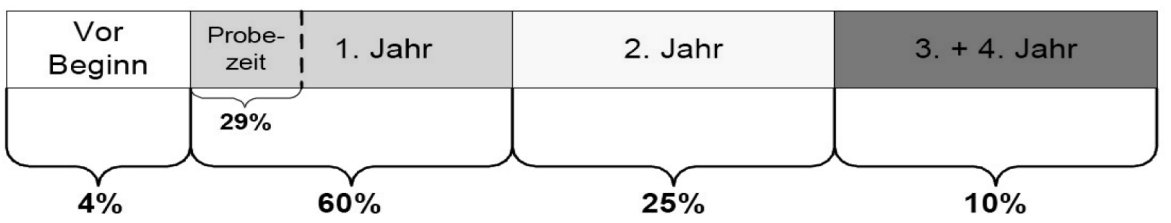

Abb. 3: Zeiträume der Vertragslösungen

der Jugendliche im Bildungssystem verbleibt und eine höhere Qualifikation anstrebt, beispielsweise ein Studium. Ein »horizontaler Abbruch « meint, dass der Jugendliche im Bildungssystem verbleibt, allerdings eine berufliche Umorientierung vornimmt, beispielsweise durch die Wahl eines anderen Ausbildungsberufs oder durch die Rückkehr in die Berufsvorbereitung. Ein »Abbruch nach unten « intendiert demgegenüber ein ersatzloser Ausstieg aus der beruflichen oder allgemeinbildenden Qualifizierung. Um »echte Abbrecher « handelt es sich somit dann, wenn begonnene formale berufliche Ausbildungen ersatzlos vor der Abschlussprüfung beendet werden, wenn auf eine weitere berufliche Ausbildung verzichtet wird, oder wenn der Jugendliche - auch nur zeitweise - den Status des An- oder Ungelernten oder Arbeitslosen innehat.

Betrachtet man sich die Zeiträume, in denen Vertragslösungen vorgenommen werden, so finden im ersten Ausbildungsjahr 60 Prozent und im zweiten Ausbildungsjahr 25 Prozent der Ausbildungsabbrüche statt (siehe Abb. 3). Obwohl die Zahl der Abbrecher mit 4 Prozent vor Be- ginn der Ausbildung eher als gering bezeichnet werden kann, ist sie aus der Sicht der Betriebe mit ihren Folgen doch groß, da zu diesem Personenkreis oftmals Jugendliche mit höherwertigen Bildungsabschlüssen, wie Abitur oder Fachhochschulreife zählen, und diese Lehrstellen meist nicht mehr adäquat besetzt werden können. Die Abbrüche im ersten Ausbildungsjahr lassen oftmals auf eine verfehlte Berufswahl mit nicht erfüllten Vorstellungen bei den Jugendlichen schließen. Hierzu kann auch eine fachliche Unteroder Überforderung innerhalb der Ausbildung führen. Im weiteren Verlauf der Ausbildung sinkt zwar das Abbruchverhalten deutlich, der entstandene wirtschaftliche, zeitliche und persönliche Schaden aufseiten der Betriebe und der Jugendlichen ist aber nicht mehr zu kompensieren.

Der Prozess, der letztlich zu einem Abbruch führt, hängt von den beteiligten Personen ab. Als sicher gilt, dass es sich in den meisten Fällen nicht um eine Ad-hoc Entscheidung handelt. So vergeht im Vorfeld eines Ausbildungsabbruchs meist ein Zeitraum von zwei Wochen bis zu acht Monaten, bis die Vertragslösung endgül- tig vorgenommen wird. Hieraus kann man schließen, dass nicht leichtfertig abgebrochen wird und eine Vorlaufzeit vorhanden ist, in der Warnsignale wahrgenommen werden müssen und somit präventive Maßnahmen eingeleitet werden können.

Verschiedene Studien (3) belegen, dass Ausbildungsabbrüche nur zu einem geringeren Teil durch fachliche Defizite der Lernenden verursacht werden. Eine Hauptursache liegt vielmehr in mangelnden »überfachlichen Kompetenzen « der Jugendlichen, die mit Berufsreife zu tun haben. Im Einzelnen sind damit soziale und personale Kompetenzen sowie Methoden-Kompetenzen gemeint. Diese überfachlichen Kompetenzen bilden nicht nur die Basis für präventive Maßnahmen während der Ausbildung, sondern auch für eine wirksame Berufsorientierung vor der Einmündung (erste Transition) sowie für den Übergang ins Erwerbsleben nach der Berufsausbildung (zweite Transition).

Neben einer Vielzahl an präventiven Maßnahmen, die im Vorfeld und im Verlauf einer Berufsausbildung, beispielsweise durch vertiefte Berufsorientierung in der Schule, Einzelberatungsgespräche für Jugendliche durch Berufsberater und Schulsozialarbeiter, Jobscouts, Ausbildungslotse, Berufs- und Studienwahlpass, Praktika, Elternabende und Elternberatung, Berufseinstiegsbegleiter, Ausbildungsbegleiter der Verbände, oder durch Case Management organisiert und durchgeführt werden können, kommt der Diagnose und Rückmeldung von überfachlichen Kompetenzen, der Messung eines potenziellen Abbruchrisikos bei Jugendlichen in der dualen Ausbildung eine zentrale Bedeutung zu. Um dies bewerkstelligen zu können, müssen Angebote von Berufsberatung, Kammern, Gewerkschaften und anderen Institutionen optimiert sowie strukturelle Probleme des Ausbildungssystems berücksichtigt werden. Insbesondere handelt es sich um folgende drei Schwerpunktbereiche:

- Verbesserung systematischer Kooperation zwischen den an der Berufsausbildung beteiligten Ausbildern, Lehrpersonen, Berufsberatern und Case Managern

- Bereitstellung erprobter und valider Instrumente zur Identifikation von Jugendlichen mit Abbruchsrisiko und problematischen überfachlichen Kompetenzen sowie 


\begin{tabular}{|l|l|l|}
\hline Methodenkompetenzen & Sozialkompetenzen & Personalkompetenzen \\
\hline - Analysefähigkeit & - Selbstständigkeit & - Motivation \\
- Reflexivität & - Kommunikationsfähigkeit & - Neugierde \\
- Flexibilität & - Kooperationsfähigkeit & - Pflichtbewusstsein \\
- zielorientiertes Handeln & - Führungsfähigkeit & - Gelassenheit \\
- Arbeitstechniken & - Konfliktfähigkeit & - Hilfsbereitschaft \\
& - situationsgerechtes Auftreten & \\
& - soziale Verantwortung & \\
\hline
\end{tabular}

Abb. 4: Dimensionen des Diagnose-Verfahrens zu überfachlichen Kompetenzen

- Implementierung von innovativen Methoden zur holistischen Beratung und Förderung dieser Jugendlichen insbesondere zur aktiven Verbesserung defizitärer Kompetenzbereiche

\section{Wie man überfachliche Kompetenzen feststellt}

Seit zwei Jahren wird am Eidgenössischen Hochschulinstitut für Berufsbildung EHB (Zollikofen, Schweiz) und an der Hochschule der Bundesagentur für Arbeit (Mannheim) ein Forschungs- und Entwicklungsprojekt zur Thematik «Überfachliche Kompetenzen und Abbruchrisiko erfassen und bewerten» durchgeführt.

Das entwickelte Diagnose-Verfahren zur Messung und Rückmeldung von überfachlichen Kompetenzen und des Abbruchrisikos trägt das Kürzel «smK+p», wobei das «s» für soziale, das «m» für methodische, das «p» für personale und das «K» für Kompetenzen steht. Die Methode «smK+p» ist ein ScreeningVerfahren, welches auf eine Beurteilungsund Auswertungsökonomie setzt und einen breiten Überblick über die wichtigsten Facetten der Handlungskompetenzen liefert. Bei Screening-Verfahren sind Ergebnisbewertung und Testökonomie besonders wichtig, was aber nicht bedeutet, dass die teststatistischen Gütekriterien nicht beachtet würden.

Das Diagnose-Verfahren misst überfachliche Kompetenzen in der Breite und meldet die Ergebnisse den Berufslernenden und Lehrpersonen in Echtzeit zurück. Das Abbruchrisiko wird durch eine Ska- la mit neun Indikatoren erfasst. Derzeit werden einige Dimensionen zu überfachlichen Kompetenzen untersucht (vgl. Abb. 4). Jeder der in der Tabelle aufgeführten Dimension (z. B. Kommunikationsfähigkeit) sind mindestens sechs Aussagen zugeordnet, die die entsprechenden Fähigkeiten, Kenntnissen oder Haltungen abbilden. Werden von einem Jugendlichen alle sechs Aussagen beantwortet, bildet der Mittelwert dieser sechs Aussagen den Wert für die Kommunikationsfähigkeit. Alle Aussagen werden auf einer sechsstufigen Skala beurteilt. Der Fokus der Beurteilungen liegt in der Anwendung der jeweiligen Fähigkeit, Kenntnis oder Haltung in beruflichen Handlungssituationen.

Das Diagnose-Verfahren besteht in seiner Papier-und-Bleistift-Fassung aus einem Buch mit Selbstbeurteilungsbögen für Jugendliche und Auswertungsbögen für den Diagnostiker. In der InternetFassung steht dem Diagnostiker ein Online-Selbstbeurteilungsbogen für Jugendliche zum Ausfüllen am PC sowie ein Online-Auswertungstool zur Verfügung. Insgesamt dauert das Ausfüllen des Selbstbeurteilungsbogens auf dem $\mathrm{Pa}$ pier oder online zwischen 30 und $45 \mathrm{Mi}$ nuten. Bei der Papierfassung werden für jede Selbstbeurteilung weitere 90 Minuten zum Auswerten und Bewerten der verschiedenen überfachlichen Kompetenzen sowie für das Erstellen der Grafiken und Tabellen benötigt. Bei der Onlinefassung wird die Auswertung und Bewertung der überfachlichen Kompetenzen von jedem einzelnen Jugendlichen von der Software automatisiert vorgenommen und binnen weniger Sekunden dem Jugendlichen und der Lehrperson in Form von Grafiken und Tabellen rückgemeldet.

Für das Einrichten der Onlineverbindung im PC-Raum für eine Klassentestung von rund 20 Jugendlichen benötigt der Diagnostiker vorab etwa eine Stunde Zeit. Vergleicht man beide Fassungen miteinander, so besitzt die Onlinefassung eine große Testökonomie, insbesondere bei der Auswertung der einzelnen Datensätze und bei der Erstellung der Grafiken und Tabellen.

\section{Zielsetzungen des Selbstbeurteilungsbogens}

Das Diagnose-Verfahren «smK+p» verfolgt verschiedene Zielsetzungen. Zum einen sollen Diagnostiker ein einfach handhabbares Diagnoseinstrument zur Messung und Bewertung von überfachlichen Kompetenzen sowie des Abbruchrisikos zur Verfügung haben, mit dessen Hilfe Ist-, Soll- und Entwicklungsprofile von Jugendlichen erstellt sowie als Grundlage für die Bestimmung eines Kompetenzentwicklungsbedarfs und zur gezielten Ableitung von Entwicklungszielen und Fördermaßnahmen herangezogen werden können. Zum anderen sollen über die Dauer einer beruflichen Ausbildung der Entwicklungsverlauf einzelner oder aller Kompetenzdimensionen bei wenigen Jugendlichen oder der ganzen Klasse aufgezeigt sowie das Abbruchrisiko bei allen Jugendlichen permanent diagnostiziert werden. 
Der «smK+p» sollte jeweils zu Beginn eines jeden Lehrjahres eingesetzt, ausgewertet und Ziele mit den Jugendlichen vereinbart und entsprechende Beratungsangebote durchgeführt werden. Kurz vor oder während der Abschlussprüfung sollte das Instrument nicht eingesetzt werden, da die gewonnenen Ergebnisse für Entwicklungsgespräche nicht mehr analysiert und aufbereitet werden können. ken an einen Abbruch nur an einem Ort fest, ohne dass es der Andere erfährt.

Vielen Jugendlichen ist es nicht möglich, sich bei Problemen aktiv um Hilfe zu bemühen. Um sie erfassen und beraten zu können, braucht es deshalb umfassende Diagnose- und Beratungsinstrumente, die allen Prozessbeteiligten zur Verfügung stehen und zum Einsatz kommen müssen. •

\section{Viele Maßnabmen greifen erst nach dem Ausbildungsabbruch«}

Die Anwendung des Diagnose-Verfahrens «smK+p» mit den entsprechenden Analysen, Interpretationen, Gesprächsvorbereitungen, Zielvereinbarungen und Fördermaßnahmen setzen den Besuch einer Weiterbildung voraus.

\section{Fazit}

Bei der Prävention von Ausbildungsabbrüchen sollten zwei Hauptansätze verfolgt werden:

- Einerseits müssen vorhandene Maßnahmen weiterentwickelt werden, die besonders auf Berufsgruppen mit hohen Lösungsquoten ausgerichtet sind. Neben der Intensivierung der Berufsorientierung, der Sensibilisierung der Ausbildungspersonen in Berufsschule, Betrieb und überbetrieblichen Kursen, den gezielten Ausbau von Fördermaßnahmen oder der Einrichtung niederschwelliger Beratung in der Schule oder in der Arbeitsagentur, müssen Diagnoseverfahren zur Messung von überfachlichen Kompetenzen und zum Abbruchsrisiko für die entsprechenden Personengruppen angeboten und durch sie zum Einsatz gelangen.

- Andererseits müssen die Bemühungen verstärkt werden, Kooperationen von Berufsberatern, Case Managern, Lehrpersonen und Ausbildern zu lancieren. Erste Anzeichen für Ausbildungsabbrüche zeigen sich oft nur an einem Lernort. Ohne eine Kooperation der Ausbildungsorte, des Case Managments und der Berufsberatung setzen sich Gedan-

\section{Anmerkungen}

(1) Jäger, D.A. (2009). Dropouts - Maßnahmen im internationalen Kontext. Panorama, 9/2009, S. 1-6.

(2) Bundesinstitut für Berufsbildung (2009). Datenreport zum Berufsbildungsbericht 2009 (http://datenreport.bibb.de).

(3) Z. B. Deuer, E. \& Ertelt, B. (2001). Früherkennung und Prävention von Ausbildungsabbrüchen. In Informationen für Beratungs- und Vermittlungsdienste (Hrsg.), Ausbildungsabbruch-Situation und Handlungsstrategien (S. 1417-1432). Nürnberg: Bundesagentur für Arbeit.

\section{Soziale Arbeit in Bewegung}

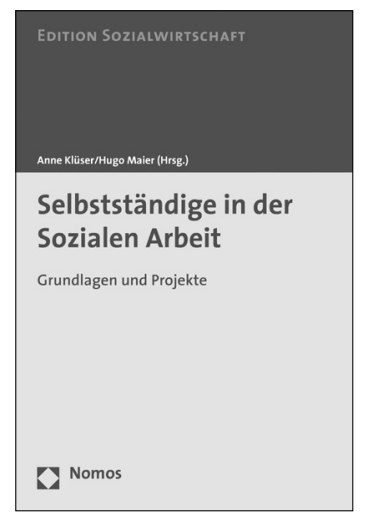

\section{Selbstständige in der Sozialen Arbeit}

Grundlagen und Projekte Herausgegeben von Dr. Anne Klüser und Prof. Dr. Hugo Maier 2009, 263 S., brosch., 44,-€, ISBN 978-3-8329-4111-6

(Edition Sozialwirtschaft, Bd. 26)

Berufliche Selbstständigkeit in der Sozialen Arbeit: Heute ist kaum noch ein Bereich denkbar, in dem dies nicht möglich wäre. In vielen Feldern Sozialer Arbeit sind Selbstständige inzwischen etabliert und zu einer festen Größe avanciert. Sie setzen neue Standards, fordern die traditionellen Wohlfahrtsverbände heraus und bringen so Bewegung in die Soziale Arbeit.

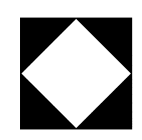

Nomos

Bitte bestellen Sie im Buchhandel oder versandkostenfrei unter $\downarrow$ www.nomos-shop.de 
„Der Verlust des Arbeitsplatzes führte bei vielen

Betroffenen zu Arbeitslosigkeit."

Aus einem Bericht der Deutschen Bank

"Bete und arbeite, sagte Benedikt von Nursia. Mache auch Arbeit zum Gebet, sagte Luther. Bete um Arbeit, sagte Pfarrer N. zum Arbeitslosen." Kurt Marti, schweizerischer Theologe und Schriftsteller (geb. 1921)

"Unsere Gesellschaft produziert viele nutzlose Dinge und im gleichen Maße auch nutzlose Menschen."

Erich Fromm, deutsch-amerikanischer Psychoanalytiker (1900-1980)

„Würde des Menschen: Nichts mehr davon, ich bitt euch. Zu essen gebt ihm, zu wohnen / Habt ihr die Blöße bedeckt,

gibt sich die Würde von selbst."

Friedrich Schiller, deutscher Dichter (1759-1805)

"Wir ham die Arbeit nur von weitem geseh'n,

und auch von weitem war sie nicht schön."

Die Drei von der Tankstelle, UFA-Film 1930

"Die Wurzel der Geschichte aber ist der arbeitende, schaffende, die Gegebenheiten umbildende und überholende Mensch.

Hat er sich erfasst und das Seine ohne Entäußerung und

Entfremdung in realer Demokratie begründet,

so entsteht in der Welt etwas, das allen in der

Kindheit scheint und worin noch niemand war: Heimat."

Ernst Bloch, deutscher Philosoph (1885-1977)

"Der Verbrecher produziert nicht nur Verbrechen,

sondern auch das Kriminalrecht und damit auch den Professor,

der Vorlesungen über das Kriminalrecht hält, und zudem das

unvermeidliche Kompendium, worin dieser selbe Professor

seine Vorträge als ,Warer auf den allgemeinen Markt wirft.

Damit tritt Vermehrung des Nationalreichtums ein."

Karl Marx, Abschweifungen über produktive Arbeit (1863) 